\title{
Out of the Box Artificial Intelligence (OBAI): The Beginning of a New Era in Artificial Intelligence
}

\author{
Satish Gajawada ${ }^{1}$, \& Hassan M. H. Mustafa ${ }^{2}$ \\ ${ }^{1}$ IIT Roorkee Alumnus, India \\ ${ }^{2}$ Banha University, Egypt \\ Correspondence: Satish Gajawada, IIT Roorkee Alumnus, India.
}

Received: January 6, 2022

Accepted: February 7, 2022

Online Published: February 14, 2022

doi:10.5539/cis.v15n2p6

URL: https://doi.org/10.5539/cis.v15n2p6

\begin{abstract}
The main purpose of writing this article is to unify all the OUT OF THE BOX ideas (under Artificial Intelligence) invented by the corresponding author of this work during the period (2013-2022) under a single umbrella titled "Out of the BOX Artificial Intelligence Field (OBAI Field)". All the OUT OF THE BOX ideas which are proposed under Artificial Intelligence will come under new field titled OBAI Field which is defined in this work. A new Artificial Intelligence field titled "Artificial Cartoon Algorithms (ACA)" is invented in this work. ACA is a sub-field of OBAI field as it is an OUT OF THE BOX idea. Four new algorithms titled "Artificial Cartoon Popeye Algorithm", "Artificial Cartoon Chhota Bheem Algorithm", “Artificial Cartoon Jerry Algorithm" and "Artificial Cartoon Happy Kid Algorithm" are designed in this work.
\end{abstract}

Keywords: out of the box, artificial intelligence, out of the box AI, AI, OBAI, cartoon algorithms, ACA, artificial cartoon popeye algorithm, artificial cartoon chhota bheem algorithm, artificial cartoon jerry algorithm, artificial cartoon happy kid algorithm

\section{Literature Review and Definition of Out of the Box Artificial Intelligence}

Artificial Human Optimization, Artificial Soul Optimization, Artificial God Optimization, Artificial Satisfaction, Deep Loving, Nature Plus Plus Inspired Computing, Artificial Heart Neural Networks, Artificial Excellence, Stories Inspired Optimization Algorithms, Artificial Intelligence Plus Plus (AI++), Twenty Second Century Artificial Intelligence, Super Artificial Neural Networks, Super Evolutionary Computing, Super Computational Intelligence, The Interesting and Complete Artificial Intelligence (ICAI) fields are OUT OF THE BOX ideas under Artificial Intelligence. Hence all these fields are part of the new field titled "Out of the Box Artificial Intelligence (OBAI)" (Satish Gajawada \& Hassan M. H. Mustafa, 2019a; Satish Gajawada \& Hassan M. H. Mustafa, 2019b; Satish Gajawada \& Hassan M. H. Mustafa, 2020a; Satish Gajawada \& Hassan M. H. Mustafa, 2020b; Satish Gajawada \& Hassan M. H. Mustafa, 2020c; Satish Gajawada \& Hassan M. H. Mustafa, 2020d; Satish Gajawada \& Hassan M. H. Mustafa, 2021a; Satish Gajawada \& Hassan M. H. Mustafa, 2021b; Satish Gajawada \& Hassan M. H. Mustafa, 2021c; Satish Gajawada \& Hassan M. H. Mustafa, 2021d; Satish Gajawada \& Hassan M. H. Mustafa, 2021e; Satish Gajawada \& Hassan M. H. Mustafa, 2021f). The new field Artificial Cartoon Algorithms (ACA) invented in this work is also the sub-field of OBAI Field.

Articles (A. Ahmadi-Javid, 2011; Ahmadi SA, 2017; Burman, R. et al., 2017; Dai C. et al., 2007; Da-Zheng Feng et al., 2015; Devika P. D et al, 2015; Edris Fattahi et al., 2018; Eita M.A. et al., 2010; Esmaeil Atashpaz-Gargari et al., 2007; Feng, X. et al., 2015; Hamid Reza Kamali et al., 2015; Hao Liu et al., 2014; Kaur, Rishemjit et al., 2013; L. M. Zhang et al., 2009; Mingyi Zhang et al., 2013; Muhammad Rizwan Tanweer et al., 2014; M. R. Tanweer et al., 2014; M.R. Tanweer et al., 2015a; M.R. Tanweer et al., 2015b; Prakasha S et al., 2013; Ruo-Li Tang et al., 2015; R.V.Rao et al., 2011; Satish Gajawada et al., 2019a; Singh M.K. et al., 2013; Sridhar N et al., 2014; Wang L. et al., 2014; Xu Y. et al., 2010) belong to Artificial Human Optimization field. Hence all these articles come under OBAI field.

\section{Artificial Cartoon Algorithms}

All Artificial Intelligence algorithms inspired from Cartoons will belong to new field titled "Artificial Cartoon Algorithms (ACA)". Fig. 1. shows cartoon character "Popeye". Fig. 2. shows cartoon character "Chhota Bheem". Fig. 3. shows cartoon character "Jerry". Fig. 4. shows cartoon character "Happy Kid". Four ACA shown in Fig. 
5., Fig. 6., Fig. 7. and Fig. 8. belong to new field "OBAI" which is created in this work.

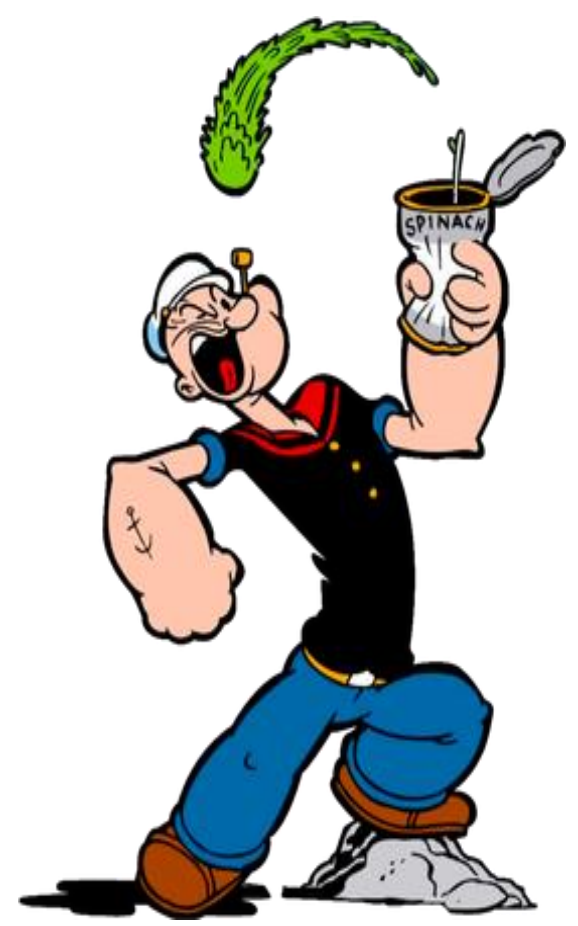

Figure 1. Popeye (with Spinach)

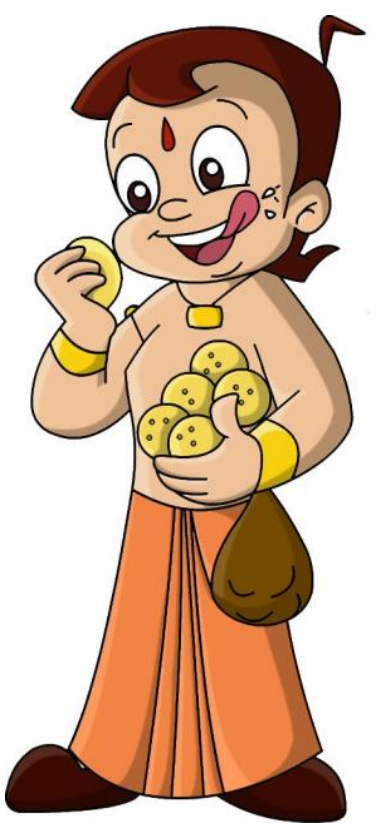

Figure 2. Chhota Bheem (with Laddu) 


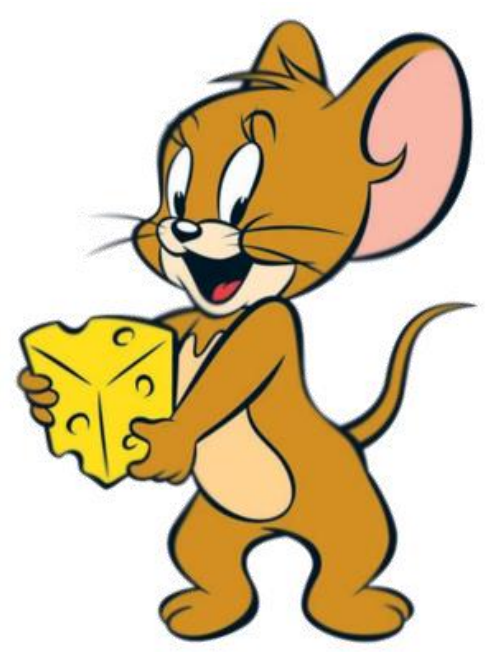

Figure 3. Jerry (with Cheese)

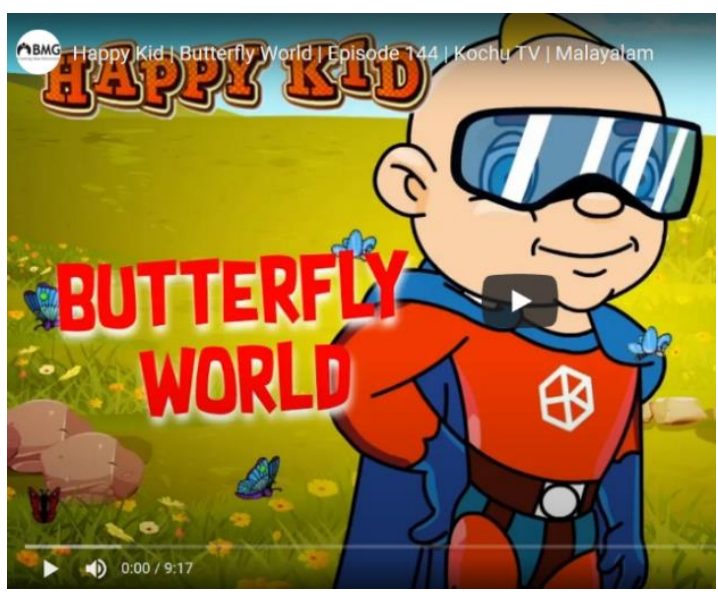

Figure 4. Happy Kid

\section{Artificial Cartoon Popeye Algorithm}

All Artificial Popeyes are initialized in line number one. In line number two, iteration count is set to zero. In line number three, local best of all Artificial Popeyes are identified. In line number four, global best of all Artificial Popeyes are identified. Based on random number generated and PopeyeSpinachProbability in line number 6, the Popeye is classified as "Popeye with Spinach" or "Popeye without Spinach". "Popeye with Spinach" eats Spinach and hence he can move in search space irrespective of anything. Based on random number generated and HelpOfPopeyeWithSpinachProbability in line number 10, "Popeye without Spinach" either receives help from "Popeye with Spinach" or not. In line numbers 11 and 12, "Popeye without Spinach" moves in search space and updates position and velocity as he receives help from "Popeye with Spinach". On the other hand, "Popeye without Spinach" is halted and doesn't do anything if he doesn't receive help from "Popeye with Spinach". This process is repeated for all the Artificial Popeyes in the population. In line number 18, the iteration count is incremented by one and the control goes to next generation. This process continues until termination condition reached is true.

1) All Artificial Popeyes are initialized

2) Generations count is initialized with zero

3) All Artificial Popeyes identify their local best

4) All Artificial Popeyes identify their global best 


\section{5) loop through for each particle do}

6) if ( random_number_generate $(0,1)<$ PopeyeSpinachProbability ) then

7) Popeye with Spinach updates Velocity

8) Popeye with Spinach updates Position

9) else // Popeye without Spinach

10) if ( random_number_generate $(0,1)<$ HelpOfPopeyeWithSpinachProbability) then

Popeye without Spinach updates Velocity

12)

Popeye without Spinach updates Position

13) else

14)

15)

16) end if

17) end for

18) generations $+=1$

19) while (termination condition not reached)

Figure 5. Artificial Cartoon Popeye Algorithm

\section{Artificial Cartoon Chhota Bheem Algorithm}

All Artificial Chhota Bheems are initialized in line number one. In line number two, iteration count is set to zero. In line number three, local best of all Artificial Chhota Bheems are identified. In line number four, global best of all Artificial Chhota Bheems are identified. Based on random number generated and ChhotaBheemLadduProbability in line number 6, the Chhota Bheem is classified as "Chhota Bheem with Laddu" or "Chhota Bheem without Laddu". "Chhota Bheem with Laddu" eats Laddu and hence he can move in search space irrespective of anything. Based on random number generated and HelpOfChhotaBheemWithLadduProbability in line number 10, "Chhota Bheem without Laddu" either receives help from "Chhota Bheem with Laddu" or not. In line numbers 11 and 12, "Chhota Bheem without Laddu" moves in search space and updates position and velocity as he receives help from "Chhota Bheem with Laddu". On the other hand, "Chhota Bheem without Laddu" is halted and doesn't do anything if he doesn't receive help from "Chhota Bheem with Laddu". This process is repeated for all the Artificial Chhota Bheems in the population. In line number 18, the iteration count is incremented by one and the control goes to next generation. This process continues until termination condition reached is true.

1) All Artificial Chhota Bheems are initialized

2) Generations count is initialized with zero

3) All Artificial Chhota Bheems identify their local best

4) All Artificial Chhota Bheems identify their global best

5) loop through for each particle do

6) if ( random_number_generate $(0,1)<$ ChhotaBheemLadduProbability ) then

7) Chhota Bheem with Laddu updates Velocity

8) Chhota Bheem with Laddu updates Position

9) else // Chhota Bheem without Laddu

10) if ( random_number_generate $(0,1)<$ HelpOfChhotaBheemWithLadduProbability) then 
15)

end if

16) end if

17) end for

18) generations $+=1$

19) while (termination condition not reached)

Figure 6. Artificial Cartoon Chhota Bheem Algorithm

\section{Artificial Cartoon Jerry Algorithm}

All Artificial Jerrys are initialized in line number one. In line number two, iteration count is set to zero. In line number three, local best of all Artificial Jerrys are identified. In line number four, global best of all Artificial Jerrys are identified. Based on random number generated and JerryCheeseProbability in line number 6 , the Jerry is classified as "Jerry with Cheese" or "Jerry without Cheese". "Jerry with Cheese" eats Cheese and hence he can move in search space irrespective of anything. Based on random number generated and HelpOfJerryWithCheeseProbability in line number 10, "Jerry without Cheese" either receives help from "Jerry with Cheese" or not. In line numbers 11 and 12, "Jerry without Cheese" moves in search space and updates position and velocity as he receives help from "Jerry with Cheese". On the other hand, "Jerry without Cheese" is halted and doesn't do anything if he doesn't receive help from "Jerry with Cheese". This process is repeated for all the Artificial Jerrys in the population. In line number 18, the iteration count is incremented by one and the control goes to next generation. This process continues until termination condition reached is true.
1) All Artificial Jerrys are initialized
2) Generations count is initialized with zero
3) All Artificial Jerrys identify their local best
4) All Artificial Jerrys identify their global best
5) loop through for each particle do
6) if ( random_number_generate $(0,1)<$ JerryCheeseProbability ) then
7) Jerry with Cheese updates Velocity
8) Jerry with Cheese updates Position
9) else // Jerry without Cheese
10) if ( random_number_generate $(0,1)<$ HelpOfJerryWithCheeseProbability) then
11)
Jerry without Cheese updates Velocity
12)
Jerry without Cheese updates Position
13) else
14)
15)
end if
16) end if
17) end for
18) generations $+=1$
19) while (termination condition not reached)

Figure 7. Artificial Cartoon Jerry Algorithm

\section{Artificial Cartoon Happy Kid Algorithm}

All Happy Kids are initialized in line number one. In line number two, iteration count is set to zero. In line number three, local best of all Artificial Happy Kids are identified. In line number four, global best of all Artificial Happy Kids are identified. Based on random number generated and HappyKidBananaProbability in line number 6, the Happy Kid is classified as "Happy Kid with Banana" or "Happy Kid without Banana". "Happy Kid with Banana" eats Banana and hence he can move in search space irrespective of anything. Based 
on random number generated and HelpOfHappyKidWithBananaProbability in line number 10, "Happy Kid without Banana" either receives help from "Happy Kid with Banana" or not. In line numbers 11 and 12, "Happy Kid without Banana" moves in search space and updates position and velocity as he receives help from "Happy Kid with Banana". On the other hand, "Happy Kid without Banana" is halted and doesn't do anything if he doesn't receive help from "Happy Kid with Banana". This process is repeated for all the Artificial Happy Kids in the population. In line number 18, the iteration count is incremented by one and the control goes to next generation. This process continues until termination condition reached is true.

1) All Artificial Happy Kids are initialized

2) Generations count is initialized with zero

3) All Artificial Happy Kids identify their local best

4) All Artificial Happy Kids identify their global best

5) loop through for each particle do

6) if ( random_number_generate $(0,1)<$ HappyKidBananaProbability ) then

7) Happy Kid with Banana updates Velocity

8) Happy Kid with Banana updates Position

9) else //

10) if ( random_number_generate $(0,1)<$ HelpOfHappyKidWithBananaProbability) then Happy Kid without Banana updates Velocity

12) Happy Kid without Banana updates Position

13) else

14)

15)

end if

16) end if

17) end for

18) generations $+=1$

19) while (termination condition not reached)

Figure 8. Artificial Cartoon Happy Kid Algorithm

\section{Opportunities in Out of the Box Artificial Intelligence Field}

According to us, there is no end to the list of the opportunities in "Out of the Box Artificial Intelligence (OBAI)" field. Some of them are listed below:

1) International Institute of OBAI, Germany

2) Indian Institute of OBAI, India

3) IBM OBAI Research Labs, Switzerland

4) Google OBAI Research Labs, USA

5) B.Tech, OBAI at IIT Mumbai

6) M.Tech, OBAI at University of Texas

7) $\mathrm{PhD}, \mathrm{OBAI}$ at University of Australia

8) PostDoc, OBAI at Harvard University

9) International Conference on OBAI, Singapore

10) International Journal on OBAI, United Kingdom

11) Out of the Box Artificial Intelligence - A New Course on Coursera

12) Seminar on OBAI, Africa

13) Book on OBAI, Elsevier Publishers 


\section{Conclusions}

A new field titled "Out of the Box Artificial Intelligence (OBAI)" is invented in this work. The main point to be noted from this work is that the Artificial Intelligence Algorithms can take inspiration from anything and anywhere. The inspiration can come from cartoons like "Popeye", "Chhota Bheem", "Jerry" and "Happy Kid" too. Another important point is that in this work Particle Swarm Optimization algorithm is modified by adding the concepts of ACA field and four new algorithms titled "Artificial Cartoon Popeye Algorithm", "Artificial Cartoon Chhota Bheem Algorithm", "Artificial Cartoon Jerry Algorithm" and "Artificial Cartoon Happy Kid Algorithm" are created. This is done for the sake of simplicity. The ideal method is to create "Artificial Cartoon Algorithms" from scratch.

\section{Acknowledgement}

Thanks to everyone (and everything) who directly or indirectly helped us to reach the stage where we are now today.

\section{Biography of Authors}

\section{Satish Gajawada}

Satish Gajawada is the Designer of new field titled "Artificial Heart Neural Networks (AHNN)". He received a SALUTE and APPRECIATION from the IEEE chair, Dr. Eng. Sattar B. Sadkhan for his numerous achievements within the field of science. His Research Project is featured by NASA Astrophysics Data System. His Research Project is indexed in AGRIS (maintained by the Food and Agriculture Organization of the United Nations (FAO)). One of his research projects has been partially funded by a research grant received from IBM Corporation as part of the IBM Shared University Research Award (IBM SUR Award). He is called "Father of Artificial Human Optimization Field" by few experts for his valuable contribution to the new field titled "Artificial Human Optimization (AHO)." He got "5 out of 5" for "Contribution to Existing Knowledge" and "Evidence Supports Conclusion" for his article "Artificial God Optimization - A Creation" published at Computer and Information Science, Canada. He received invitation for a fully-funded Summer INTERNSHIP project in 2009 from Telecom Sud Paris, Cedex, France. He is the Creator of "Smile Gita - The Song of Smile" approved by Social Science Research Network, Elsevier. He is the Creator of new branches under or related to Artificial Intelligence like Artificial Satisfaction, Deep Loving and Nature++ Inspired Computing. He coined the terms under Artificial Intelligence like "Artificial Human Optimization", "Artificial Soul Optimization" and "Artificial God Optimization". Search the phrase "father of Artificial Human Optimization" on "Google Search Engine" and it displays content related to him. He is the Program Committee Member of Artificial Intelligence Conference "SCAI2021". He published 50+ publications. He is the Creator of new branches titled "Artificial Excellence", "Stories Inspired Optimization Algorithms" and "Artificial Intelligence Plus Plus (AI++)". He is the author of book "Twenty Second Century Artificial Intelligence". He is the inventor of new AI branch titled "The Interesting and Complete Artificial Intelligence (ICAI)". He is the Founder and Father of new field titled "Out of the Box Artificial Intelligence (OBAI)". He got selected as "International Best Researcher" at INSO 2021 Scientist Awards.

\section{Hassan M. H. Mustafa}

Department of Educational Technology, Banha University, Egypt. He was born in Cairo, on first of October 1947. He received his B.Sc. Degree and M.Sc. Degrees in Electrical Engineering from Military Technical College Cairo-Egypt in 1970, and1983 respectively. He received his Ph.D. degree at Computer Engineering and Systems in 1996 from Ain Shams University -Faculty of Engineering Cairo-Egypt. Currently, he is an Associate Professor with Computer Engineering, Department, Al-Baha University K.S.A. He is a member with a set of Scientific, Engineering, and educational technology Societies such as IIIS (International Institute of Informatics and Systemics), the Society of Digital Information and Wireless Communications (SDIWC). And at the International Association of Online Engineering IAOE. He is a senior member at International Economics Development Research Center (IEDRC) organization. Furthermore, he has been appointed as a member of technical comity for Artificial Neural Networks research work at IASTED organization during the period (2009-2012). He is one of advisors with ELIXIR Journal and he has been appointed as a reviewer member at WCSIT Journal. His fields of interest include Artificial Neural Networks, Natural Inspired Computations, and their applications for simulation, modelling and evaluation of learning processes /phenomena. Recently, he has been nominated as the Grand-father of AHO according to Satish Gajawada the founder of AHO. He is an author / co-author for more than 150 published papers \& technical reports \& books. All articles have been published at international specialized conferences and journals during time period from 1983 till 2021. 


\section{References}

A. Ahmadi-Javid (2011). Anarchic Society Optimization: A human-inspired method. Proc. 2011 IEEE Congr. Evol. Comput., pp. 2586-2592. https://doi.org/10.1109/CEC.2011.5949940

Ahmadi, S. A. (2017). Neural Comput \& Applic, 28(Suppl 1), 233. https://doi.org/10.1007/s00521-016-2334-4

Burman, R., Chakrabarti, S., \& Das (2017). S. Soft Comput, 21, 3267. https://doi.org/10.1007/s00500-015-2007-8

Dai, C., Zhu, Y., \& Chen, W. (2007). Seeker Optimization Algorithm. In Wang Y., Cheung Y., Liu H. (Eds)., Computational Intelligence and Security, Lecture Notes in Computer Science, 4456, Springer, Berlin, Heidelberg. https://doi.org/10.1007/978-3-540-74377-4_18

Da-Zheng, F., Han-Zhe, F., \& Hai-Qin, Z. (2015). Human Behavior Algorithms for Highly Efficient Global Optimization. Retrieved from https://arxiv.org/ftp/arxiv/papers/1507/1507.04718.pdf

Devika, P. D., \& Dinesh, P. A, Rama Krishna Prasad, Manoj Kumar Singh (2015). ASBO Based Compositional in Combinatorial Catalyst. J. Math.Comput.Sci., 5(3), 351-393.

Edris Fattahi, Mahdi Bidar, and Hamidreza Rashidy Kanan (2018). Int. J. Comp. Intel. Appl. 17, 1850002. https://doi.org/10.1142/S1469026818500025

Eita, M. A., \& Fahmy, M. M. (2010). Group Counseling Optimization: A Novel Approach. In Bramer M., Ellis R., Petridis M. (Eds.), Research and Development in Intelligent Systems XXVI, Springer, London. https://doi.org/10.1007/978-1-84882-983-1_14

Esmaeil, A. G., \& Caro, L. (2007). Imperialist competitive algorithm: An algorithm for optimization inspired by imperialistic competition. IEEE Congress on Evolutionary Computation.

Feng, X., Zou, R., \& Yu, H. (2015). Soft Comput, 19, 2955. https://doi.org/10.1007/s00500-014-1459-6

Hamid, R. K., Ahmad, S., Mohammad, A. V. Z., \& Hassan, K. Z. (2015). Immigrant Population Search Algorithm for Solving Constrained Optimization Problems. Applied Artificial Intelligence, 29(3), 243-258. https://doi.org/10.1080/08839514.2015.1004613

Hao, L., Gang, X., Gui-yan, D., \& Yu-bo, S. (2014). Human Behavior-Based Particle Swarm Optimization. The Scientific World Journal, Article ID 194706, 14 pages. https://doi.org/10.1155/2014/194706

Kaur, R., Kumar, R., Bhondekar, A. P., \& Kapur, P. (2013). Human opinion dynamics: An inspiration to solve complex optimization problems. Scientific Reports, pp. 1-7. ISSN 2045-2322. https://doi.org/10.1038/srep03008

Muhammad, R. T., \& Suresh, S. (2014). Human cognition inspired particle swarm optimization algorithm. IEEE Ninth International Conference on Intelligent Sensors, Sensor Networks and Information Processing (ISSNIP).

Prakasha, S., Shashidhar, H. R., Manoj, K. S., \& Raju, G. T. (2013). Clustering of Text Document based on ASBO. Wulfenia journal, 20(6), 152-165.

Rao, R. V., Savsani, V. J., \& Vakharia, D. P. (2011). Teaching-learning-based optimization: A novel method for constrained mechanical design optimization problems. Computer-Aided Design, 43(3), 303-315. https://doi.org/10.1016/j.cad.2010.12.015

Satish, G., \& Hassan, M. H. M. (2019a). Ten Artificial Human Optimization Algorithms. Transactions on Machine Learning and Artificial Intelligence, United Kingdom, 7(3), 1-16. https://doi.org/10.14738/tmlai.73.6631

Satish, G., \& Hassan, M. H. M. (2019b). Artificial Soul Optimization - An Invention. Transactions on Machine Learning and Artificial Intelligence, United Kingdom, 7(5), 36-44. https://doi.org/10.14738/tmlai.75.7322

Satish, G., \& Hassan, M. H. M. (2020a). Artificial God Optimization - A Creation. Computer and Information Science, Canada, 13(1), 41-50. https://doi.org/10.5539/cis.v13n1p41

Satish, G., \& Hassan, M. H. M. (2020b). Artificial Satisfaction - The Brother of Artificial Intelligence. Global Journal of Computer Science and Technology, 20(1-D), 29-36. https://doi.org/10.34257/GJCSTDVOL20IS1PG29

Satish, G., \& Hassan, M. H. M. (2020c). Deep Loving - The Friend of Deep Learning. Global Journal of Computer Science and Technology, 20(1-D), 51-53. https://doi.org/10.34257/GJCSTDVOL20IS1PG51 
Satish, G., \& Hassan, M. H. M. (2020d). Nature Plus Plus Inspired Computing - The Superset of Nature Inspired Computing. Global Journal of Computer Science and Technology, 20(1-C), 53-55. https://doi.org/10.34257/GJCSTCVOL20IS1PG53

Satish, G., \& Hassan, M. H. M. (2021a). Artificial Heart Neural Networks. Twenty Second Century Artificial Intelligence, 48-50. https://doi.org/10.9734/bpi/mono/978-93-5547-146-8/CH7

Satish, G., \& Hassan, M. H. M. (2021b). Artificial Excellence. Twenty Second Century Artificial Intelligence, 51-57. https://doi.org/10.9734/bpi/mono/978-93-5547-146-8/CH8

Satish, G., \& Hassan, M. H. M. (2021c). Stories Inspired Optimization Algorithms. Twenty Second Century Artificial Intelligence, 58-59. https://doi.org/10.9734/bpi/mono/978-93-5547-146-8/CH9

Satish, G., \& Hassan, M. H. M. (2021d). AI++: Artificial Intelligence Plus Plus. Transactions on Machine Learning and Artificial Intelligence, United Kingdom, 9(4), 15-17. https://doi.org/10.14738/tmlai.94.10592

Satish, G., \& Hassan, M. H. M. (2021e). Twenty Second Century Artificial Intelligence. ISBN 978-93-5547-146-8 (Print). ISBN 978-93-5547-147-5 (eBook). B P International. Retrieved from https://ssrn.com/abstract=3937288

Satish, G., \& Hassan, M. H. M. (2021f). The Interesting and Complete Artificial Intelligence (ICAI) - Version 1. Transactions on Machine Learning and Artificial Intelligence, United Kingdom, 9(6), 20-25. https://doi.org/10.14738/tmlai.96.11195

Singh, M. K. (2013). A New Optimization Method Based on Adaptive Social Behavior: ASBO. In: Kumar M. A., R. S., Kumar T. (Eds.), Proceedings of International Conference on Advances in Computing, Advances in Intelligent Systems and Computing, pp. 174. https://doi.org/10.1007/978-81-322-0740-5_98

Sridhar, N., Nagaraj, R., \& Manoj, K. S. (2014). PID Controller Auto tuning using ASBO Technique. Journal of Control Engineering and Technology, 4(3), 192-204. https://doi.org/10.14511/jcet.2014.040305

Tang, R. L., \& Fang, Y. J. (2015). Modification of particle swarm optimization with human simulated property. Neurocomputing, 153, 319-331. https://doi.org/10.1016/j.neucom.2014.11.015

Tanweer, M. R., Suresh, S., \& Sundararajan, N. (2014). Human meta-cognition inspired collaborative search algorithm for optimization. International Conference on Multisensor Fusion and Information Integration for Intelligent Systems (MFI), pp. 1-6. https://doi.org/10.1109/MFI.2014.6997631

Tanweer, M. R., Suresh, S., \& Sundararajan, N. (2015a). Self regulating particle swarm optimization algorithm. Information Sciences: an International Journal, 294(C), 182-202. https://doi.org/10.1016/j.ins.2014.09.053

Tanweer, M. R., Suresh, S., \& Sundararajan, N. (2015b). Improved SRPSO algorithm for solving CEC 2015 computationally expensive numerical optimization problems. IEEE Congress on Evolutionary Computation (CEC), pp. 1943-1949. https://doi.org/10.1109/CEC.2015.7257123

Wang, L., Ni, H., Yang, R., Fei, M., \& Ye, W. (2014). A Simple Human Learning Optimization Algorithm. In Fei M., Peng C., Su Z., Song Y., Han Q. (Eds.), Computational Intelligence, Networked Systems and Their Applications, Communications in Computer and Information Science, 462, Springer, Berlin, Heidelberg. https://doi.org/10.1007/978-3-662-45261-5_7

Xu, Y., Cui, Z., \& Zeng, J. (2010). Social Emotional Optimization Algorithm for Nonlinear Constrained Optimization Problems. In Panigrahi, B. K., Das, S., Suganthan, P. N., Dash, S. S. (Eds.), Swarm, Evolutionary, and Memetic Computing, Lecture Notes in Computer Science, 6466, Springer, Berlin, Heidelberg. https://doi.org/10.1007/978-3-642-17563-3_68

Zhang, L. M., Dahlmann, C., \& Zhang, Y. (2009). Human-inspired algorithms for continuous function optimization. In IEEE International Conference on Intelligent Computing and Intelligent Systems, 1, 318-321. https://doi.org/10.1109/ICICISYS.2009.5357838

Zhang, M. Y., Zhang, L., \& Yan, Q. (2013). The Human-Inspired Algorithm: A Hybrid Nature-Inspired Approach to Optimizing Continuous Functions with Constraints. Journal of Computational Intelligence and Electronic Systems, 2(1), 80-87. https://doi.org/10.1166/jcies.2013.1039

\section{Copyrights}

Copyright for this article is retained by the author(s), with first publication rights granted to the journal.

This is an open-access article distributed under the terms and conditions of the Creative Commons Attribution license (http://creativecommons.org/licenses/by/4.0/). 